\title{
Canada's ability to respond to a national health crisis hampered by jurisdictional issues, untested emergency plans
}

$\mathrm{A}$ s a high school student in Israel, Dr. Daniel Kollek volunteered for ambulance duty, helping to provide basic life support services. "I was present at terrorist bombings. It colours the way you look at the world," says the associate professor of emergency medicine at McMaster University in Hamilton, Ontario.

Israel's emergency health response is, of course, highly developed (CMAJ 2003;169[11]:1198) and it is glaringly obvious to Kollek, who obtained his MD from the University of Ottawa in Ottawa, Ont., and later did emergency training in Israel, that Canada's disaster and emergency preparedness pales by comparison. There is "such an opportunity" to be better prepared, he argues.

He's not alone. Most observers say Canada has significantly improved its capacity to handle health emergencies and disasters since the outbreak of severe acute respiratory syndrome (SARS) in 2003, in large measure because that outbreak forced parties to talk to one another. "The silver lining of SARS was that it brought people together to plan, and when we started planning, everyone knew each other," says Dr. Ross Upshur, associate scientist at the Sunnybrook Health Sciences Centre in Toronto, Ont.

But Upshur and others add that much more needs to be done, as evidenced by the scathing report Emergency Preparedness in Canada: How the fine arts of bafflegab and procrastination hobble the people who will be trying to save you when things get really bad..., published in September, 2008, by the Standing Senate Committee on National Security and Defence.

Key findings included evidence of poor collaboration among federal, provincial and municipal governments; lack of sharing of lessons learned and best practices; and lack of training funds for municipalities.

The Senate report was about overall

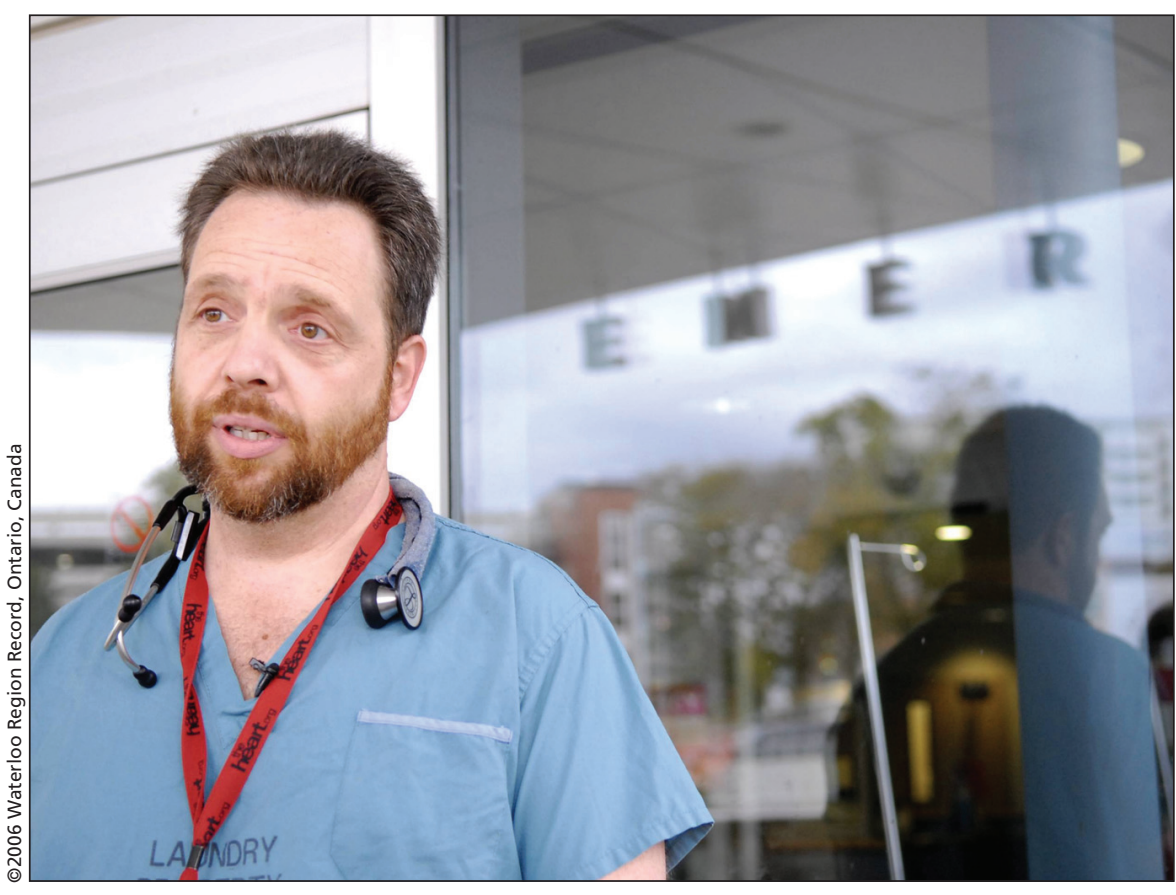

Dr. Daniel Kollek says Canada has "such an opportunity" to improve preparedness for the health impacts of disasters and emergencies.

preparedness but there typically are health consequences in every form of emergency, whether flood, ice storm, forest fire or terrorist attack with mass casualties.

Canada had an opportunity to test its preparedness for an influenza outbreak this spring, as all countries grappled with the possibility of a global pandemic stemming from the $\mathrm{A}(\mathrm{H} 1 \mathrm{~N} 1)$ swine flu outbreak in Mexico (www.cmaj.ca /earlyreleases/090805.pdf). Pandemic plans, experts agree, are the most highly developed and certainly the best funded of Canada's health emergency response plans. Roughly $\$ 1$ billion has been directed toward Canadian pandemic planning. But a great deal of effort is also going toward strengthening an "all hazards" approach — "generic" planning that can be adapted to deal with the health implications of any disaster or epidemic.

Canada faces political, geographic, cultural and manpower challenges in all its health emergency response planning, but at issue is the extent to which top-level planning is in sync with frontline responders, since most emergencies begin locally and response is typically from the bottom up.

"Unless the front line gets organized within itself, any plan that is organized by upper levels of government may not be applicable or practical," argues Kollek. He is adamant about the need to establish a standard of care for dealing with the health aspects of disasters and emergencies based on best evidence and developed by a multidisciplinary team of experts.

That conviction prompted him to help establish the Centre for Excellence in Emergency Preparedness, a nonprofit group. While Kollek's main concern is ensuring that hospitals and emergency wards are better prepared to deal with the hike in demand associated 
with events having mass casualties, he says it is important that the planning go beyond individual facilities.

In fact, health emergency plans with detailed responsibility charts and committees with unwieldy acronyms abound. But critics say too many plans are untested, personnel are often untrained and interjurisdictional training exercises are rare. That's in part because sustaining interest in emergency preparedness and obtaining funding between emergencies can be difficult.

Yet systems "can't just sit there," says Andrew Graham, adjunct professor of the school of policy studies at Queen's University in Kingston, Ont. "They have to be tested again and again."

As always, a key obstacle to any form of national planning in Canada is the fact that health care is a provincial responsibility and intergovernmental cooperation is invariably a challenge. As well, levels of preparedness differ across the country, yet emergency response systems need to be linked,

Indeed, even Canada's ability to meet its obligations under the International Health Regulations adopted by the United Nations' World Health Assembly is in doubt because of the federal government's limited powers. Key intergovernmental agreements about public health surveillance have not been finalized, yet the regulations oblige nations to report public health emergencies within 24 hours. "Right now, the federal government can't even demand information from the provinces," notes Dr. Kumanan Wilson, Canada Research Chair in Public Health Policy at the University of Ottawa.

"Every jurisdiction has their own emergency management system," acknowledges Dr. Theresa Tam, director general of the Centre for Emergency Preparedness and Response at the Public Health Agency of Canada. "But there is definitely a need for a national leadership and coordination role."

That need is particularly acute in a pandemic situation when geopolitical boundaries are rendered meaningless. "We want at least a baseline of agreedupon approaches so [for example] people are not using vaccines or antivirals differently," says Tam.
While Public Safety Canada is the lead federal body for dealing with emergencies and disasters, Tam's centre is responsible for coordinating the overall federal health response to emergencies. But the fact that it is located within the Public Health Agency of Canada has caused some confusion.

Meanwhile, emergency management as a discipline, based on evidence-based practice, "is only just emerging in this country," according to an article in Health Canada's April 2000 health policy research bulletin on emergency management (www.healthcanada.gc.ca /hpr-bulletin).

And when emergency planners and health care personnel sit down to plan, their terminology and culture are often radically different. Tam acknowledges that she herself is "on a learning curve" when it comes to the meshing of health and emergency management.

Yet a common understanding is key, says Upshur, who is also director of the Joint Centre for Bioethics at the University of Toronto. In the midst of Hurricane Katrina, doctors and firefighters in New Orleans, Louisiana, were insisting on different protocols for evacuating patients from hospitals, he says.

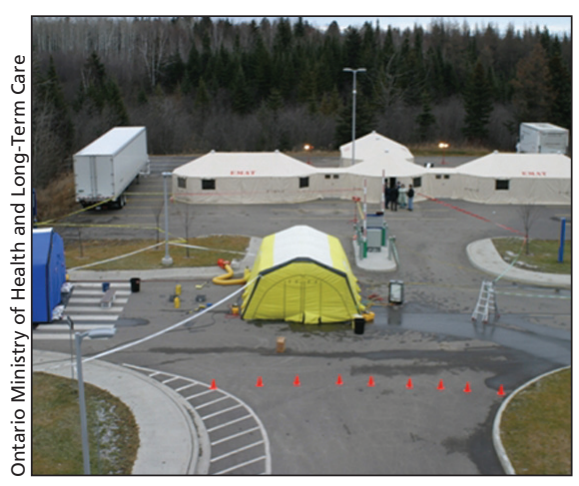

Ontario's Emergency Medical Assistance Team was deployed to Thunder Bay, Ont., in late 2008 as part of a larger provincial exercise to test readiness to respond to severe weather and critical infrastructure failure. Pictured above is the team's mobile hospital.

Importantly, emergency management operates on a top-down, command-and-control basis, while health care decision-making is largely decentralized. This creates vulnerabilities for health care, such as a potential lack of clarity regarding leadership, "since leadership is driven by level, and emergencies could be at the municipal, provincial or federal level," says Graham. As well, it is not evident how well the health care sector can "reach outside the silo," such as seeking help with transport from the military during an emergency, he adds. "The greatest failure in emergency planning is "the cold call to set up a system in the middle of an emergency."

Harmonizing protocols within the health care system is also an issue. An Institute for Clinical Evaluative Studies study of hospital admissions during the SARS outbreak in Toronto stressed the need for hospitals to adopt "uniform clinical urgency criteria" to avoid nonurgent admissions (Healthc Q. 2009;12 [1]:30-32).

To Kollek, key issues include advance testing of plans and surge capacity. He notes that Israeli preparations include equipping hospital hallways with oxygen and electrical outlets.

An abiding issue for planners is that people trust and go to their family physician in an emergency, but engaging family physicians in emergency planning is "a tough nut to crack," says Kollek. Adds Upshur: "We train doctors to be independent. Maybe we ought to start training them to be part of an emergency response team."

In terms of concrete achievements, Tam points to the creation and ongoing testing of 24/7 contact points for communicating during a health emergency. "Even though one might think that is a small step ... [this] is something we haven't done before. We relied on the fact that everybody knows each other in Canada and chief medical officers of health have each other on speed dial."

Meanwhile, federal, provincial and territorial governments are "very close" to agreeing on a Pan Canadian Health Emergency Management System that identifies roles and responsibilities, Tam says. "When you look at the amount of difference that exists in this country in different provincial systems, in fact it is a major commitment to move ahead towards more consistency." — Ann Silversides, CMAJ

DOI:10.1503/cmaj.090870 